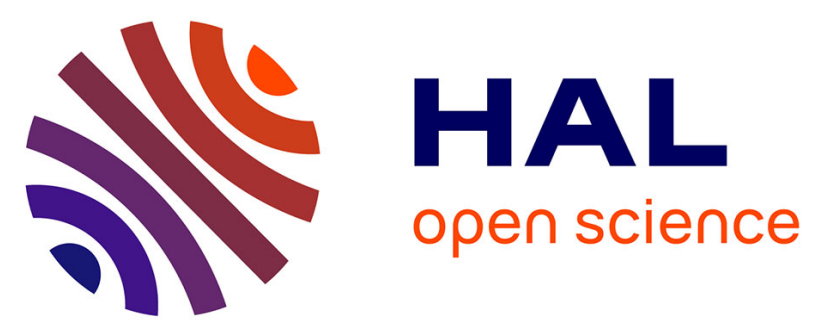

\title{
First clinical inflammatory demyelinating events of the central nervous system in a population aged over 70 years: A multicentre study
}

Noëlle Lavandier, Mickaël Bonnan, Clarisse Carra-Dallière, Mahmoud Charif, Pierre Labauge, Jean-Philippe Camdessanché, Gilles Edan, Aurore Naudin, David Brassat, Jonathan Ciron, et al.

\section{To cite this version:}

Noëlle Lavandier, Mickaël Bonnan, Clarisse Carra-Dallière, Mahmoud Charif, Pierre Labauge, et al.. First clinical inflammatory demyelinating events of the central nervous system in a population aged over 70 years: A multicentre study. Multiple Sclerosis and Related Disorders, 2019, 28, pp.309-312. 10.1016/j.msard.2018.12.016 . hal-02928079

\section{HAL Id: hal-02928079 \\ https://hal.umontpellier.fr/hal-02928079}

Submitted on 21 Oct 2021

HAL is a multi-disciplinary open access archive for the deposit and dissemination of scientific research documents, whether they are published or not. The documents may come from teaching and research institutions in France or abroad, or from public or private research centers.
L'archive ouverte pluridisciplinaire HAL, est destinée au dépôt et à la diffusion de documents scientifiques de niveau recherche, publiés ou non, émanant des établissements d'enseignement et de recherche français ou étrangers, des laboratoires publics ou privés.

\section{(ㄷ)(1) $\$$}

Distributed under a Creative Commons Attribution - NonCommerciall 4.0 International 


\section{ABSTRACT}

\section{Background}

Few data are available regarding patients with very late-onset inflammatory demyelinating events. (VLO-IDE).

\section{Objectives}

The aim of this study was to describe the clinical, biological, and radiological characteristics and aetiological diagnosis of very late first inflammatory demyelinating events of the central nervous system.

\section{Methods}

We conducted a national descriptive retrospective multicentre study on a case series of patients aged $>70$ years at the time of VLO-IDE. Patients were recruited from a national call on behalf of the 'Société Francophone de la Sclérose en Plaques' (French Multiple Sclerosis Society).

\section{Results}

Twenty-five patients were referred (F:M sex ratio 2.1:1). The most frequent clinical impairment was a spinal cord deficit (23/25), usually severe (disability score, median EDSS $4,7[2-9,5]$. Spinal cord lesions were usually extensive, spanning at least three segments $(11 / 25)$, and large brain lesions were also observed (lesions $>20 \mathrm{~mm}$ in 6/25). The final aetiological diagnoses comprised multiple sclerosis $(9 / 25)$, neuromyelitis optica spectrum disorders (7/25), neurosystemic lupus erythematosus (2/25), transverse myelitis without aetiological diagnosis (6/25) and optic neuritis (1/25).

\section{Conclusions}

This study highlights a particular phenotype of first clinical inflammatory demyelinating events in predominantly female patients aged $>70$ years who have severe motor impairment with common longitudinal extensive myelitis and large and common very active radiological inflammatory lesions. Neuromyelitis optica spectrum disorders seem overrepresented.

\section{Keywords:}

Multiple sclerosis, optic neuritis, transverse myelitis, neuromyelitis optica, late onset, ageing

\section{INTRODUCTION}

A late-onset clinical inflammatory demyelinating event (LO-IDE) of the central nervous system is usually defined as an onset after age 50. Late-onset clinically isolated syndrome (LO-CIS), multiple sclerosis (LO-MS) or neuromyelitis optica (LO-NMO) have a low prevalence ${ }^{1,2}$. However, their incidence sharply decreases with age and they rarely occur after age 70. The goal of this study was to examine very late-onset inflammatory demyelinating events (VLO-IDE) occurring in elderly patients ( $\geq 70$ years).

\section{METHODS}

\section{Study design}

We conducted a national descriptive retrospective multicentre study from a national call on behalf of Société Francophone de la Sclérose en Plaques (SFSEP), the French national MS society. Eligible patients were identified through a national call to French neurologists involved in MS. Eight French MS centres participated in the study. Patients gave written consent through the French Observatory of Multiple Sclerosis (OFSEP) project. 
Inclusion criteria were as follows: 70-year-old patients or older with a first clinical inflammatory demyelinating event of the central nervous system. Exclusion criteria were as follows: neoplastic, paraneoplastic, or infectious inflammatory lesions; prior history (onset before 70 years) of clinical symptoms suggestive of inflammatory neurological event.

Medical records were reviewed retrospectively and data were collected for demographic details (sex, age of onset), biological results including CSF, main clinical features, final diagnosis, and treatment. Brain and spinal cord MRI were reviewed. Final diagnosis was made using the most recent criteria for MS, NMO, CIS and lupus erythematosus (LE) ${ }^{3-5}$ Medical records were reviewed retrospectively and data were collected for demographic details. Expanded Disability Status Scale (EDSS) was obtained during relapse.

\section{RESULTS}

\section{Population}

Thirty-one patients were collected from clinicians. Six patients were excluded: 5 with insufficient clinical/MRI data to fulfil the inclusion/exclusion criteria and one who was 69 years old. Finally, 25 patients were included (Table 1), 17 were female (F:M sex ratio 2.1:1). The median follow-up was 13.5 months [5-72].

\section{Final diagnosis}

Among the 25 patients, 9 (36\%) fulfilled both the 2005 and 2010 McDonald diagnostic ${ }^{5}$ criteria for MS, $4(16 \%)$ with relapsing-remitting (RR) MS and 5 (20\%) with primary progressive (PP) MS. Seven (28\%) patients fulfilled the latest NMO Spectrum Disorder (NMOSD, 2015) criteria $^{4}$, and two of them fulfilled the NMO (2006) criteria. Two patients $(8 \%)$ fulfilled criteria for $\mathrm{LE}^{3}$, revealed by extended myelitis or by cognitive impairment and another patient had transverse myelitis with APS and erythema elevatum diutinum (EED). In addition, 5 (20\%) had a clinically isolated syndrome: transverse myelitis and an optic neuritis in 4 and 1 patients, respectively. One patient had atypical transverse myelitis and cortical blindness with negative investigation.

\section{Clinical characteristics}

Motor impairment was the prominent phenotype (20/25) at the beginning and 23/25 during the course of evolution, with para- or tetraparesis $(8 / 25,1 / 25$ at the beginning and $6 / 25,5 / 25$, during the course of evolution). Motor impairment was often serious (median EDSS 4.7[2$9,5]$ ) with frequent vesico-sphincter disorders (15/25). Optic neuritis was less common $(2 / 25)$. Five of 9 patients with MS had primary progressive clinical impairment and 4/9 had acute relapses.

Atypical clinical presentations expressed by cortical signs were found in several patients: visual extinction (patient 1 with MS diagnosis and a very large brain lesion), anosognosia with cortical blindness and confusion (patient 21 with serious transverse myelitis) also with ischaemic lesions on brain MRI, aphasia (patient 17 with LE) and acute cognitive disorder (patient 18 with LE).

Seven patients with NMOSD had spinal cord lesions, 5 with prominent severe motor impairment and 2 with sensitive myelitis. Disability was severe in these NMOSD patients (median EDSS: 7 [4,5-8,5]).

\section{Biological findings}

Antinuclear antibodies were found with a high level in the serum of 2/2 patients with LE and in one patient with transverse myelitis and intrathecal IgG synthesis (\#23). APS were found in patients with TM and skin vasculitis (EED). AQP4 were positive in 4/7 and MOG in 1/7 
patients with NMOSD. Intrathecal IgG synthesis (IgG index $>0.7$ or oligoclonal bands) was found in $60 \%$ of all cases.

\section{MRI features}

Spinal cord lesions were common (92\%) with extensive lesions spanning at least three segments in $44 \%$ of them. Inflammatory brain lesions were observed in $68 \%$ and large brain lesions (>20mm) were observed in 4 MS and 1 NMOSD patients (Fig. 1). Brain lesion load was mild in LE patients, although these patients presented severe clinical signs.

\section{Treatment}

All patients initially received intravenous high-dose methylprednisolone. Several diseasemodifying drugs were administered (see Table1). Severe drug side-effects were observed in only one case.

\section{DISCUSSION}

Most studies dedicated to LO-IDE included patients aged over 50 years and most of these patients were usually less than 70 years old ${ }^{1,6-8}$. Therefore, data are limited in the elderly and our cohort is the first to our knowledge to target VLO-IDE. Mean age at first neurological event was 76.2 years with an overrepresentation of females. Whatever the final diagnosis, CIS occurring at a very late age appears to have specific characteristics compared to those occurring in younger patients: spinal cord involvement predominates (92\%), clinical impairment is often motor and severe with longitudinally extensive myelitis, and MRI lesions are usually extended (large brain lesions $>20 \mathrm{~mm}$ : 24\%; spinal cord lesions $>3$ levels: $44 \%$ ).

Prevalence of LO-MS is lower than $2 \%{ }^{6,9}$. Although prevalence cannot be derived from our data, the few VLO-CIS patients we gathered from this nationwide study suggest a very low prevalence. The predominance of spinal cord involvement and severe motor impairment is in keeping with LO-MS features ${ }^{6,8,9}$. Interestingly, although LO-MS are often progressive ${ }^{8,9}$, real RRMS with clinical and radiological activity may still occur in the elderly.

Although 7 VLO-CIS patients remained unclassified at the end of the study, the main finding is the over-representation of non-MS disorders in this cohort of VLO-IDE, especially the NMOSD ratio of 2.5:1, as compared with 50:1 in younger subjects ${ }^{2,9,10}$.

Ischemic attacks are common in older adults. This study shows however that our cases are different: progressive clinical onset over several days or months and usually not sudden and provides evidence supporting inflammation in all our 25 cases on MRI, including at the level of the spinal cord: not focused on anterior cords, ovoid sharply delineated morphology and /or presence of contrast enhancement supporting inflammation. However a broad ischemic and non ischemic differential diagnosis should be considered. ${ }^{11}$

The main limitation of the study is its retrospective design from a French national MS society call. In addition, most patients were seen in tertiary MS referral centres, so there may have been a referral bias of atypical cases. However, clinicians should keep in mind that although inflammatory and demyelinating disorders are mostly associated with young age, they might occur in the elderly either as severe CIS, true MS or mostly as NMOSD.

\section{Table 1. Main characteristics of VLO-IDE cohort}

Figure 1. Brain and spinal cord MRI of VLO-IDE. A. Brainstem attack in NMOSD AQP4(-) (\#12, longitudinally extensive myelitis not shown). B. Large lesions $>20 \mathrm{~mm}(\# 4$, RR-MS). C and D. Longitudinally extensive myelitis in VLO-CIS TM (\#21). E. Multiple spinal cord lesions in NMOSD AQP4(+) (\#11, optic neuritis not shown). F. Large lesions (\#1, 
RR-MS). G. Large lesion (\#5, PP-MS). MRI sequences: brain slices: FLAIR weighted imaging (WI), inset and C: T1-enhanced WI; D, E: T2 WI.

Acknowledgment. We are indebted to Ray Cooke for copyediting.

Disclosures. Commission Nationale Informatique et Libertés (CNIL) approved the Observatory of Multiple Sclerosis (OFSEP) project.

Contributors. NL MB and JCO contributed to the writing of the manuscript. NL, JCO, CCD, $\mathrm{MC}, \mathrm{PL}, \mathrm{JPC}, \mathrm{GE}, \mathrm{AN}, \mathrm{DB}, \mathrm{JC}, \mathrm{PC}, \mathrm{MB}, \mathrm{CD}, \mathrm{AM}$ and $\mathrm{BB}$ contributed to reviewing the manuscript, the recruitment of patients and data collection.

Competing interest. No competing interest in relation with the manuscript.

Funding. No funding in relation with the manuscript.

\section{REFERENCES}

1.Bermel RA, Rae-Grant AD and Fox RJ. 2010.Diagnosing multiple sclerosis at a later age: more than just progressive myelopathy. Multiple sclerosis. 16: 1335-40.

2.Krumbholz M, Hofstadt-van Oy U, Angstwurm K, Kleiter I, Jarius S, Paul F, Aktas O, Buchholz G, Kern P, Straube A, Kümpfel T.2015.Very late-onset neuromyelitis optica spectrum disorder beyond the age of 75. Journal of neurology. 262: 1379-84.

3.Hochberg MC. 1997.Updating the American College of Rheumatology revised criteria for the classification of systemic lupus erythematosus. Arthritis and rheumatism. 40: 1725.

4.Wingerchuk DM, Banwell B, Bennett JL, Cabre P, Carroll W, Chitnis T, de Seze J, Fujihara K, Greenberg B, Jacob A, Jarius S, Lana-Peixoto M, Levy M, Simon JH, Tenembaum S,Traboulsee AL, Waters P, Wellik KE, Weinshenker BG; International Panel for NMO Diagnosis.2015. International consensus diagnostic criteria for neuromyelitis optica spectrum disorders. Neurology. 85: 177-89.

5.Polman CH, Reingold SC, Banwell B, Clanet M, Cohen JA, Filippi M, Fujihara K, Havrdova E, Hutchinson M, Kappos L, Lublin FD, Montalban X, O'Connor P, SandbergWollheim M, Thompson AJ, Waubant E, Weinshenker B, Wolinsky JS. 2011. Diagnostic criteria for multiple sclerosis: 2010 revisions to the McDonald criteria. Annals of neurology. 69: 292-302.

6.Bove RM, Healy B, Augustine A, Musallam A, Gholipour T and Chitnis T. 2012. Effect of gender on late-onset multiple sclerosis. Multiple sclerosis. 18: 1472-9.

7.Delalande S, De Seze J, Ferriby D, Stojkovic T and Vermersch P. 2002. Late onset multiple sclerosis. Revue neurologique. 158: 1082-7.

8.Sanai SA, Saini V, Benedict RH, Zivadinov R, Teter BE, Ramanathan M, WeinstockGuttman B.2016. Aging and multiple sclerosis. Multiple sclerosis. 22: 717-25.

9.Collongues N, Marignier R, Jacob A, Leite MI,Siva A, Paul F, Zephir H, Akman-Demir G, Elsone L, Jarius S, Papeix C, Mutch K, Saip S, Wildemann B, Kitley J, Karabudak R, Aktas O, Kuscu D, A Altintas A, Palace J, Confavreux C and De Seze J. 2014. Characterization of neuromyelitis optica and neuromyelitis optica spectrum disorder patients with a late onset. Multiple sclerosis. 20: 1086-94. 
10.Collongues N, Papeix C, Zephir H, Audoin B, Cotton F, Durand-Dubief F, Vukusic S, Brassat D, Laplaud D, Marignier R; Groupe Nomadmus.2014. Nosology and etiologies of acute longitudinally extensive transverse myelitis. Revue neurologique. 170: 6-12.

11. Weidauer, S., Wagner, M., Nichtweiß, M. 2017. Magnetic Resonance Imaging and Clinical Features in Acute and Subacute Myelopathies. Clin. Neuroradiol. 27: 417-433. 
Table 1. Main characteristics of VLO-IDE cohort

\begin{tabular}{|c|c|c|c|c|c|c|c|c|c|c|}
\hline$\#$ & $\begin{array}{c}\text { Sex, } \\
\text { age at } \\
\text { onset }\end{array}$ & $\begin{array}{c}\text { Follo } \\
\text { w-up } \\
\text { (mon } \\
\text { ths) }\end{array}$ & $\begin{array}{c}\text { ED } \\
\text { SS } \\
\text { nad } \\
\text { ir }\end{array}$ & $\begin{array}{c}\text { Installa } \\
\text { tion } \\
\text { mode }\end{array}$ & Clinics & $\begin{array}{l}\text { MRI } \\
\text { Swant } \\
\text { on } \\
\text { criteri } \\
\text { a }\end{array}$ & $\begin{array}{c}\text { MR } \\
\text { I, } \\
\text { larg } \\
\text { e } \\
\text { lesio } \\
\text { n }\end{array}$ & Biology ${ }^{a}$ & $\begin{array}{c}\text { Treatm } \\
\text { ents } b\end{array}$ & $\begin{array}{c}\text { Final } \\
\text { diagnos } \\
\text { is }\end{array}$ \\
\hline 1 & $F, 84$ & 22 & 7.0 & $\begin{array}{l}\text { acute } \\
\text { (10d) }\end{array}$ & $\begin{array}{l}\text { ataxia, visual } \\
\text { extinction and } \\
\text { paraparesis }\end{array}$ & + & LBL & $\mathrm{CSF}(+)$ & $\begin{array}{l}\text { AZA, } \\
\text { MMF }\end{array}$ & RRMS \\
\hline 2 & $F, 73$ & 68 & 3.0 & - & $\begin{array}{l}\text { ON then } \\
\text { moderate } \\
\text { myelitis }\end{array}$ & + & - & CSF(+) & DMF & RRMS \\
\hline 3 & $F, 75$ & 24 & 2.0 & $\begin{array}{l}\text { acute } \\
\text { (30d) }\end{array}$ & $\begin{array}{c}\text { sensory facial } \\
\text { impairment } \\
\text { then } \\
\text { brainstem } \\
\text { syndrome } \\
\text { with } \\
\text { hemiparesis }\end{array}$ & + & LBL & CSF(+) & $\begin{array}{l}\text { IFN, } \\
\text { fingo }\end{array}$ & RRMS \\
\hline 4 & M, 74 & 15 & 5.0 & $\begin{array}{c}\text { progr. } \\
(1 \mathrm{y})\end{array}$ & $\begin{array}{c}\text { hemiparesis } \\
\text { and cerebellar } \\
\text { ataxia }\end{array}$ & + & LBL & $\mathrm{CSF}(+)$ & $\begin{array}{l}\text { NTZ, } \\
\text { steroids }\end{array}$ & RRMS \\
\hline 5 & M, 75 & 48 & 4.0 & $\begin{array}{l}\text { progr. } \\
(2 \mathrm{y})\end{array}$ & hemiparesis & + & LBL & $\mathrm{CSF}(+)$ & MTX & PPMS \\
\hline 6 & M, 70 & 40 & 7.0 & $\begin{array}{l}\text { progr. } \\
(>2 \mathrm{y})\end{array}$ & $\begin{array}{c}\text { severe } \\
\text { myelitis }\end{array}$ & + & $\begin{array}{l}\text { LE } \\
M \\
\end{array}$ & CSF(+) & none & PPMS \\
\hline 7 & F, 72 & $72 \dagger$ & 9.5 & $\begin{array}{l}\text { progr. } \\
(2 \mathrm{y})\end{array}$ & $\begin{array}{l}\text { severe } \\
\text { myelitis }\end{array}$ & + & - & $\mathrm{CSF}(+)$ & none & PPMS \\
\hline 8 & $\mathrm{~F}, \mathbf{7 0}$ & 29 & 7.5 & $\begin{array}{c}\text { progr. } \\
(4 y)\end{array}$ & $\begin{array}{c}\text { severe } \\
\text { tetraparesis }\end{array}$ & + & $\begin{array}{l}\text { LE } \\
M\end{array}$ & $\mathrm{CSF}(+)$ & none & PPMS \\
\hline 9 & M, 84 & 72 & 5 & $\begin{array}{l}\text { progr } \\
(1 \mathrm{y})\end{array}$ & $\begin{array}{c}\text { monoparesia } \\
\text { and ataxia }\end{array}$ & + & LBL & CSF(-) & none & PPMS \\
\hline $\begin{array}{l}1 \\
0\end{array}$ & $F, 76$ & $60 \dagger$ & 8.5 & $\begin{array}{c}\text { progr. } \\
(3 y)\end{array}$ & $\begin{array}{c}\text { severe } \\
\text { tetraparesis }\end{array}$ & - & $\begin{array}{l}\mathrm{LE} \\
\mathrm{M}\end{array}$ & $\begin{array}{c}\text { CSF(-), } \\
\text { AQP4(+) }\end{array}$ & $\begin{array}{l}\text { CPP, } \\
\text { ritux }\end{array}$ & $\begin{array}{c}\text { NMOS } \\
\text { D }\end{array}$ \\
\hline $\begin{array}{l}1 \\
1\end{array}$ & $\mathrm{~F}, 71$ & 71 & 7.0 & $\begin{array}{l}\text { acute } \\
(8 d)\end{array}$ & $\begin{array}{c}\text { severe } \\
\text { myelitis with } \\
\text { ON }\end{array}$ & - & $\begin{array}{l}\text { LE } \\
\text { M }\end{array}$ & $\begin{array}{c}\text { CSF(+), } \\
\text { AQP4(+) }\end{array}$ & CPP & $\begin{array}{c}\text { NMOS } \\
\text { D }\end{array}$ \\
\hline $\begin{array}{l}1 \\
2\end{array}$ & $F, 75$ & 27 & 8.0 & acute & $\begin{array}{c}\text { severe } \\
\text { tetraparesis } \\
\text { with } \\
\text { brainstem } \\
\text { syndrome }\end{array}$ & + & $\begin{array}{l}\text { LE } \\
\text { M, } \\
\text { LBL }\end{array}$ & $\begin{array}{c}\text { CSF(-), } \\
\text { AQP4(-) }\end{array}$ & $\begin{array}{l}\text { PLEX, } \\
\text { mitox, } \\
\text { MMF }\end{array}$ & $\begin{array}{c}\text { NMOS } \\
\text { D }\end{array}$ \\
\hline $\begin{array}{l}1 \\
3 \\
\end{array}$ & M, 71 & 24 & 4.0 & $\begin{array}{l}\text { acute } \\
\text { (2d) }\end{array}$ & $\begin{array}{c}\text { moderate } \\
\text { myelitis }\end{array}$ & - & $\begin{array}{l}\mathrm{LE} \\
\mathrm{M} \\
\end{array}$ & $\begin{array}{c}\text { CSF(+), } \\
\text { AQP4(+) }\end{array}$ & MMF & $\begin{array}{c}\text { NMOS } \\
\text { D }\end{array}$ \\
\hline $\begin{array}{l}1 \\
4 \\
\end{array}$ & $F, 81$ & 13 & 8.0 & $\begin{array}{l}\text { acute } \\
(3 d)\end{array}$ & severe TM & - & $\begin{array}{l}\mathrm{LE} \\
\mathrm{M} \\
\end{array}$ & $\begin{array}{c}\text { CSF(-), } \\
\text { AQP4(+) }\end{array}$ & $\begin{array}{l}\text { PLEX } \\
\text { MMF } \\
\end{array}$ & $\begin{array}{c}\text { NMOS } \\
\text { D }\end{array}$ \\
\hline
\end{tabular}




\begin{tabular}{|c|c|c|c|c|c|c|c|c|c|c|}
\hline $\begin{array}{l}1 \\
5\end{array}$ & $\mathrm{~F}, 72$ & 5 & 4,5 & $\begin{array}{l}\text { acute } \\
(2 \mathrm{~d})\end{array}$ & severe TM & - & $\begin{array}{c}\mathrm{LE} \\
\mathrm{M}\end{array}$ & $\begin{array}{c}\mathrm{CSF}(-) \\
\mathrm{AQP} 4(+)\end{array}$ & $\begin{array}{l}\text { PLEX } \\
\text { ritux }\end{array}$ & $\begin{array}{c}\text { NMOS } \\
\text { D }\end{array}$ \\
\hline $\begin{array}{l}1 \\
6\end{array}$ & M, 77 & 60 & 7 & $\begin{array}{l}\text { progr. } \\
(2 \mathrm{~m})\end{array}$ & severe TM & - & $\begin{array}{l}\text { LE } \\
M\end{array}$ & $\begin{array}{c}\mathrm{CSF}(-) \\
\operatorname{MOG}(+)\end{array}$ & $\begin{array}{c}\text { PLEX } \\
\text { ritux }\end{array}$ & $\begin{array}{c}\text { NMOS } \\
\text { D }\end{array}$ \\
\hline $\begin{array}{l}1 \\
7\end{array}$ & M, 92 & 7 & $\mathrm{n} / \mathrm{a}$ & $\begin{array}{l}\text { acute } \\
\text { (15d) }\end{array}$ & $\begin{array}{l}\text { aphasia and } \\
\text { paraparesis }\end{array}$ & - & $\begin{array}{l}\text { LE } \\
M\end{array}$ & CSF(-) & $\begin{array}{c}\text { warfari } \\
\text { ne, } \\
\text { AMD }\end{array}$ & LE \\
\hline $\begin{array}{l}1 \\
8\end{array}$ & $\mathrm{~F}, 72$ & 10 & $\mathrm{n} / \mathrm{a}$ & acute & $\begin{array}{l}\text { cognitive then } \\
\text { psychiatric }\end{array}$ & - & - & CSF(-) & $\begin{array}{c}\text { IVIG, } \\
\text { warfari } \\
\text { ne, } \\
\text { aspirine }\end{array}$ & $\begin{array}{c}\text { LE, SS, } \\
\text { APS }\end{array}$ \\
\hline $\begin{array}{l}1 \\
9\end{array}$ & $\mathrm{~F}, \mathbf{8 0}$ & 10 & 5,5 & progr. & $\begin{array}{c}\text { moderate } \\
\text { myelitis }\end{array}$ & - & - & $\mathrm{CSF}(+)$ & none & $\begin{array}{c}\text { APS, } \\
\text { skin } \\
\text { vasculiti } \\
\text { s (EED) }\end{array}$ \\
\hline $\begin{array}{l}2 \\
0\end{array}$ & M, 75 & 7 & $\mathrm{n} / \mathrm{a}$ & $\begin{array}{l}\text { acute } \\
(3 \mathrm{~d})\end{array}$ & $\begin{array}{l}\text { sensitive } \\
\text { myelitis }\end{array}$ & - & - & $\begin{array}{l}\text { CSF(+), } \\
\text { AQP4(-) }\end{array}$ & none & $\begin{array}{c}\text { CIS, } \\
\text { partial } \\
\text { TM }\end{array}$ \\
\hline $\begin{array}{l}2 \\
1\end{array}$ & F, 79 & 3 & $\mathrm{n} / \mathrm{a}$ & $\begin{array}{l}\text { acute } \\
\text { (1d) }\end{array}$ & $\begin{array}{l}\text { tetraparesis } \\
\text { and cortical } \\
\text { blindness }\end{array}$ & - & - & $\begin{array}{c}\text { CSF(-), } \\
\text { AQP4(-) }\end{array}$ & $\begin{array}{c}\text { PLEX, } \\
\text { IVIG }\end{array}$ & $\begin{array}{l}\text { CIS, } \\
\text { TM }\end{array}$ \\
\hline $\begin{array}{l}2 \\
2\end{array}$ & F, 73 & 50 & $\mathrm{n} / \mathrm{a}$ & $\begin{array}{l}\text { acute } \\
(12 d)\end{array}$ & $\begin{array}{l}\text { moderate } \\
\text { myelitis }\end{array}$ & - & - & $\begin{array}{l}\text { CSF(+), } \\
\text { AQP4(-) }\end{array}$ & PLEX & $\begin{array}{c}\text { CIS, } \\
\text { partial } \\
\text { TM }\end{array}$ \\
\hline $\begin{array}{l}2 \\
3\end{array}$ & F, 81 & 12 & 7 & $\begin{array}{l}\text { acute } \\
\text { (1d) }\end{array}$ & $\begin{array}{l}\text { severe partial } \\
\text { myelitis }\end{array}$ & - & $\begin{array}{l}\text { LE } \\
M\end{array}$ & $\mathrm{CSF}(+)$ & none & $\begin{array}{c}\text { CIS, } \\
\text { partial } \\
\text { TM }\end{array}$ \\
\hline $\begin{array}{l}2 \\
4\end{array}$ & F, 77 & 22 & 3 & acute & $\begin{array}{c}\text { moderate } \\
\text { myelitis }\end{array}$ & - & - & CSF(-) & None & $\begin{array}{c}\text { CIS, } \\
\text { TM } \\
\text { (cone) }\end{array}$ \\
\hline $\begin{array}{l}2 \\
5\end{array}$ & F, 75 & 5 & 2.5 & $\begin{array}{l}\text { acute } \\
(7 d)\end{array}$ & $\mathrm{ON}$ & - & $\begin{array}{l}\mathrm{LE} \\
\mathrm{M}\end{array}$ & $\mathrm{CSF}(+)$ & none & $\begin{array}{l}\text { CIS, } \\
\text { ON }\end{array}$ \\
\hline
\end{tabular}

${ }^{\mathrm{a}} \mathrm{CSF}(+)$ : oligoclonal banding patterns 2 and $3 ;^{\mathrm{b}}$ all attacks were treated by steroids; $\uparrow$ death. AMD: antimalarial drug; APS: antiphospholipid syndrome; AZA: azathioprine; CPP: cyclophosphamide; DMF: dimethyl fumarate; fingo: fingolimod; EED: Erythema elevatum diutinum; IFN: interferons; IVIG: intravenous immunoglobulins; LBL: large brain lesion $(>2 \mathrm{~cm})$; LE: lupus erythematosus; mitox: mitoxantrone; LEM: longitudinally extended myelitis (>3 spinal segments); MMF: mycophenolate mofetil; MTX: methotrexate; NTZ: natalizumab; PLEX: plasma exchange; ritux: rituximab; SS: Sjögren syndrome. Installation mode: acute or progressive in days (d) or years (y). 


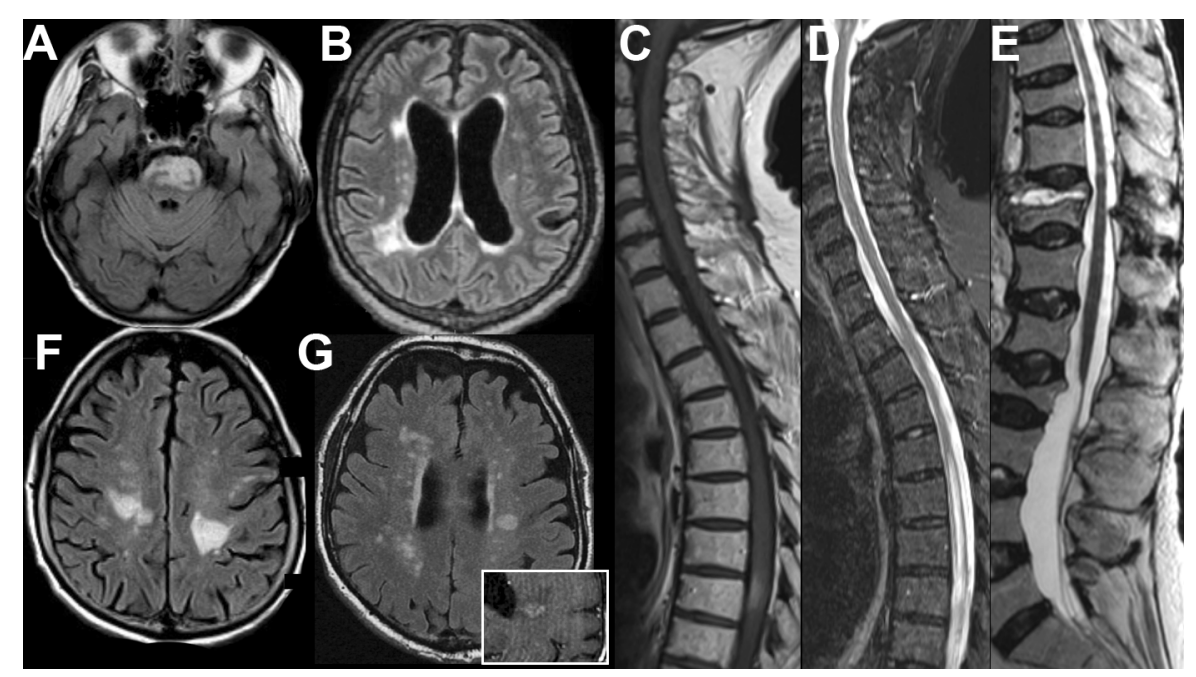

